\title{
Pushing the limit of instrument capabilities
}

\section{Denis V. Shulyak ${ }^{1}$, Werner W. Weiss ${ }^{2}$, Gautier Mathys ${ }^{3}$, Laurent Eyer $^{4}$, Alexander F. Kholtygin ${ }^{5}$, Oleg Kochukhov ${ }^{6}$, Pierre North ${ }^{7}$, Sergey N. Fabrika ${ }^{8}$ \& Tatyana E. Burlakova ${ }^{8}$}

\author{
${ }^{1}$ Institute of Astrophysics, Georg-August-University, \\ Friedrich-Hund-Platz 1, D-37077 Göttingen, Germany \\ email: denis@phys.uni-goettingen.de \\ ${ }^{2}$ Institut für Astronomie, Universität Wien, \\ Türkenschanzstraße 17, 1180 Wien, Austria \\ email: werner.weiss@univie.ac.at \\ ${ }^{3}$ European Southern Observatory, \\ Casilla 19001, Santiago 19, Chile \\ email: gmathys@eso.org \\ ${ }^{4}$ Observatoire de Genève, \\ 51 ch. des Maillettes, CH-1290 Sauverny, Switzerland \\ email: Laurent.Eyer@unige.ch \\ ${ }^{5}$ Astronomical Institute, St. Petersburg State University, \\ Universitetskii pr. 28, St. Petersburg, 198504, Russia \\ email: afkholtygin@gmail.com \\ ${ }^{6}$ Department of Physics and Astronomy, Uppsala University, \\ Box 515, 751 20, Uppsala, Sweden \\ email: Oleg.Kochukhov@fysast.uu.se \\ ${ }^{7}$ Ecole Polytechnique Fédérale de Lausanne, \\ 1015 Lausanne, Switzerland \\ email: pierre.north@epfl.ch \\ ${ }^{8}$ Special Astrophysical Observatory, Russian Academy of Sciences, \\ Nizhnii Arkhyz, Karachai Cherkess Republic, 369167, Russia
}

\begin{abstract}
Chemically Peculiar (CP) stars have been the subject of systematic research for more than 50 years. With the discovery of pulsation of some of the cool CP stars, and the availability of advanced spectropolarimetric instrumentation and high signal-to-noise, high resolution spectroscopy, a new era of CP star research emerged about 20 years ago. Together with the success in ground-based observations, new space projects are developed that will greatly benefit future investigations of these unique objects. In this contribution we will give an overview of some interesting results obtained recently from ground-based observations and discuss the future outstanding Gaia space mission and its impact on CP star research.
\end{abstract}

Keywords. stars: chemically peculiar, stars: atmospheres, stars: variables: roAp, stars: oscillations, space vehicles: instruments, Gaia mission

\section{Brief overview on CP stars}

Back in 1897, more than one century ago, the first peculiar stars were found in the course of the Henry Draper Memorial classification work at Harvard by Antonia Maury and Annie Cannon. Maury used the designation "peculiar" for the first time to describe spectral features in the remarks to the spectrum of $\alpha^{2}$ CVn, Pickering \& Maury, 1897, marking a first attempt for two-dimensional classification system considering the strength and the width of the spectral lines. 
In 1974 Preston proposed the division of main-sequence CP stars into four groups according to their spectroscopic characteristics: Preston, 1974: CP1 (Am/Fm stars), CP2 ( $\mathrm{Si}$, SrCrEu stars), CP3 (HgMn stars), CP4 (He-weak stars). More detailed spectroscopic consideration of CP stars required introduction of new subtypes of CP stars, such as Herich and $\lambda$ Boo stars. CP2 stars, including Bp/Ap, host strong surface magnetic fields (Lantz, 1993) that are likely stable on large time-intervals (North, 1983).

Abundance peculiarities were measured using the curve-of-growth method based on simple assumptions about formation of absorption lines (models of SchusterSchwarzschild, Unsöld, Milne-Eddington). Since that time and with development of new high-resolution, high signal-to-noise CCD based spectrometers, big progress has been made in abundance analysis of CP stars, revealing the presence of vertical, Ryabchikova et al., 2006, Ryabchikova et al., 2005, Ryabchikova et al., 2002, Kochukhov et al., 2006, and horizontal, Khokhlova et al., 1997, Kochukhov et al., 2004, Lüftinger et al., 2009, elements separation in their atmospheres caused by the processes of microscopic particle diffusion, Michaud, 1970.

The discovery of strong stellar surface magnetic fields Babcock, 1958 opened a new research field in astrophysics - stellar magnetism. A 200 Gauss accuracy of the magnetic field detection usually obtained with photographic plates has increased to $\approx 1$ Gauss with modern spectropolarimetry and new techniques (such as Least Square Deconvolution, or LSD, for example) - Donati et al., 1997, Wade et al., 2000.

With the discovery by D. Kurtz (Kurtz, 1978) of a 12 min pulsation period in HD 101065 a subgroup of the cool CP stars, the so-called rapidly oscillating Ap (roAp) stars, became extremely promising targets for asteroseismology, a most powerful tool for testing theories of stellar structure. Driving of the oscillations results from a subtle energy balance depending directly on the interaction between the magnetic field, convection, pulsations, and atomic diffusion. Amazing insights in the 3-D structure of stellar atmospheres became available; see, for example, Kochukhov, 2006, Freyhammer et al., 2009 .

\section{Selected results from recent ground-based observations of CP stars}

Current ground-based observations of CP stars reveal many interesting findings that require new modelling approaches and interpretations.

Accurate high-resolution observations are needed to understand the properties and origin of so-called hyper-velocity stars (HVS). These are B-type stars with peculiar galactic rest frame velocity and enhanced $\alpha$ elements and normal (solar) Fe abundances in their atmospheres. The origin of these stars is not well understood: one hypothesis is that they originate from the dynamical interaction of binary stars with the supermassive black hole in the Galactic Centre (GC), which accelerates one component of the binary to beyond the Galactic escape velocity. So far, however, no HVS has been unambiguously related to a GC origin. Determination of the place of ejection of a HVS requires the determination of space motion (accurate proper motions) and chemical composition, however, the later is hard to use to constrain their origin; see Przybilla et al., 2008. On the other hand, if $\mathrm{GC}$ origin can be proved by such future astrometric missions like Gaia (see below in this contribution) then it can bring important constraints on magnetic fields in GC region and on the formation and evolution of CP stars in general.

An important issue is raised by rotational braking observed recently for magnetic Bp star HD 37776. It was shown that this star increased its rotational period by $17.7 \mathrm{~s}$ over the past 31 years (Mikulášek et al., 2008). This can not be explained by light-time effect caused, for example, by the presence of a secondary companion which is not observed 
in radial velocity measurements. Also, the hypothesis of free-body precession due to magnetic distortion is incompatible with light curve shapes unchanged in 31 years. The plausible scenario left is a continuous momentum loss due to magnetic braking that requires magnetically confined stellar wind, which naturally can be present in HD 37776 .

Interferometry is becoming a very powerful tool in modern astrophysics since it allows for direct and model independent measurement of sizes of stellar objects. Recently, there was a report of the first detailed interferometric study of a roAp star $\alpha$ Cir (Bruntt et al., 2008). The authors used observations from the Sydney University Stellar Interferometer (SUSI) and additional data from visual and UV observations calibrated to absolute units to accurately derive $T_{\text {eff }}$ and $\log (g)$ of the star. Even thought the interferometry can provide us with accurate radii of stars, the accuracy of $T_{\text {eff }}$ determination is still limited by the incompleteness of observations in all spectral ranges (that would give a value of the bolometric flux) and model atmospheres used; see Kochukhov et al., 2009. Application of interferometric techniques to another $\mathrm{Ap}$ star $\beta \mathrm{CrB}$ is reported in this conference (JD04).

Interesting results were obtained for the well known Ap star $\epsilon$ UMa which appeared to host a brown dwarf companion. This star shows substantial variations of radial velocities measured from different spectral lines (Woszczyk \& Jasinski, 1980) which allowed to derive the parameters of the system and to infer the mass of the secondary component of $M_{2}=14.7 M_{\text {Jupiter }}$ (Sokolov, 2008).

There have been an extensive discussions over past years about the LiI $6708 \AA$ line which is usually used for the determination of $\mathrm{Li}$ abundance in magnetic Ap stars. However, due to unknown and yet unconfirmed blending with possible lines of rare-earth elements (REE) it was not definitely clear if the abundance determination suffer from systematic uncertainties or not. Recently Kochukhov, 2008 confirmed Li identification in magnetic Ap stars using detailed calculations of Zeeman pattern in Paschen-Back regime. No correlation between $\mathrm{Li}$ and REE line strengths was found thus ruling out the suspicion that the observed feature is due to an unidentified REE line. However the origin of $\mathrm{Li}$ still has to be explained theoretically by diffusion calculations and/or abundance spots on stellar surface.

High-resolution, high signal-to-noise observations allow to study not only element stratification in atmospheres of CP stars with great detail, but also the separation of different isotopes of a given element as demonstrated by Cowley et al., 2009 who studied ${ }^{40} \mathrm{Ca} /{ }^{48} \mathrm{Ca}$ isotopic anomaly in atmosphere of selected Ap stars. This was also investigated in Ryabchikova et al., 2008 who analysed calcium isotopes stratification profiles in three stars 10 Aql, HR 1217 and HD 122970 concluding that the heavy isotope concentrated towards the higher layers. Interestingly, they found no correlation in ${ }^{48} \mathrm{Ca}$ excess in atmospheres of roAp and noAp stars with the magnetic field strength.

New results of searching for the line profile variability (LPV) in the spectra of OB stars have been recently reported based on observations made with the 1.8-m telescope of Korean Bohyunsan Optical Astronomical Observatory and 6-m telescopes of Special Astrophysical Observatory, Russia. For all program stars regular and often coherent LPV was reported for all spectral lines, as shown in Fig. 1 (right panel) for the B1 supergiant $\rho$ Leo. This coherence is connected with the presence of the stellar magnetic field. The moderate dipole magnetic field of $\rho$ Leo with the polar field strength $B_{p} \approx 250 \mathrm{G}$ was detected by Kholtygin et al., 2007.

Together with the regular LPV the numerous local details of line profiles in spectra of a few program stars are detected and are connected with the formation and destruction of the small-scale structures (clumps or clouds) in the stellar wind. The evidence that numerous clumps exist in the winds of the O6 star $\lambda$ Ori A and O9.5 star $\delta$ Ori A was 

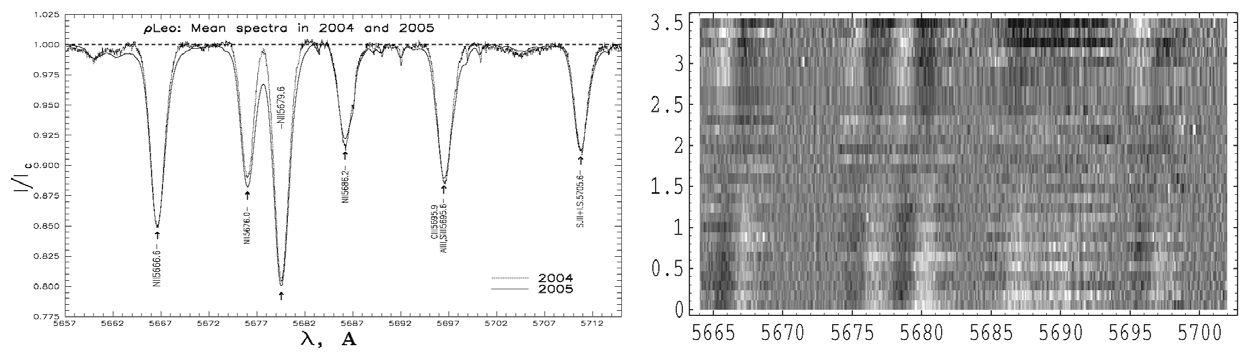

Figure 1. Left Panel: Mean spectra of $\rho$ Leo in the spectral region $5667-5715 \AA$ in 2004 and 2005. Right Panel: dynamical spectra of LPV in the same region.

found. The dynamical wavelet spectra of LPV technique is used by Kholtygin, 2008 to determine the distribution of the line fluxes for the clump ensemble in the winds of these stars.

Many new interesting results have been obtained in the past couple of years from ground-based observations of CP stars, that lead to refinements of "traditional" analyses. Based on observations of ever increasing quality we see effects that had not been previously detected. Definitely, "new" observational techniques (such as interferometry) have a large potential. Additional observational constraints will force us to re-think some of our "well-established" views on the nature, origin and evolution of CP stars bringing bridges between them and some topics of present-day astrophysics (galactic center, lowest-mass stars, etc.).

\section{Gaia space mission and CP stars}

In parallel to the progress in ground-based observations, modern space missions open a number of possibilities for CP stars' research not only in the solar neighbourhood, but also much further beyond. Scheduled to be launched in 2012, Gaia is one of the cornerstone astrometric missions of the European Space Agency (ESA) which will allow the study of the general properties and characteristics of a huge sample of stars of all spectral types. Gaia will measure position, distances, space motions, radial velocities and fundamental parameters of about 1 billion(!) objects. This scanning satellite will observe every single object (galaxies, quasars, solar system objects, etc.) to 20th magnitude, thus providing important scientific constraints for nearly all fields of modern astrophysics. With the expected astrometric accuracy of about $7 \mu$ as at 10th magnitude, Gaia's precision is more than 100 times higher than those of previously developed missions like Tycho and Hipparcos. Gaia has two telescopes with two viewing directions separated by $106.5^{\circ}$ : each telescope has a SiC primary mirror $1.45 \times 0.5 \mathrm{~m}^{2}$ and $35 \mathrm{~m}$ focal length. The key idea of Gaia design is that the images from both telescopes are combined in one focal plane which hosts a number of CCD detectors with the total size of $4500 \times 1966$ pixels. Further detailed information can be retrieved from the official Gaia website (http://www.rssd.esa.int/index.php?project=GAIA\&page=index).

Although Gaia is primarily an astrometric mission, it will also provide us with broadband spectrophotometric data in a wide spectral range. This is done by two photometers called Blue Photometer (BP) and Red Photometer (RP) with the working wavelength ranges of $330-680 \mathrm{~nm}$ and $640-1000 \mathrm{~nm}$ respectively. In addition, Gaia will be armed with a spectrograph for the radial velocity measurements (RVS) which operates in a narrow spectral range of $847-874 \mathrm{~nm}$ (around CaII infrared triplet) with the resolution $R=11500$. Every detected object will automatically pass through $\mathrm{BP} / \mathrm{RP}$ and then RVS CCD's, and this opens a wide range of possibilities for stellar studies. Indeed, RP/BP 

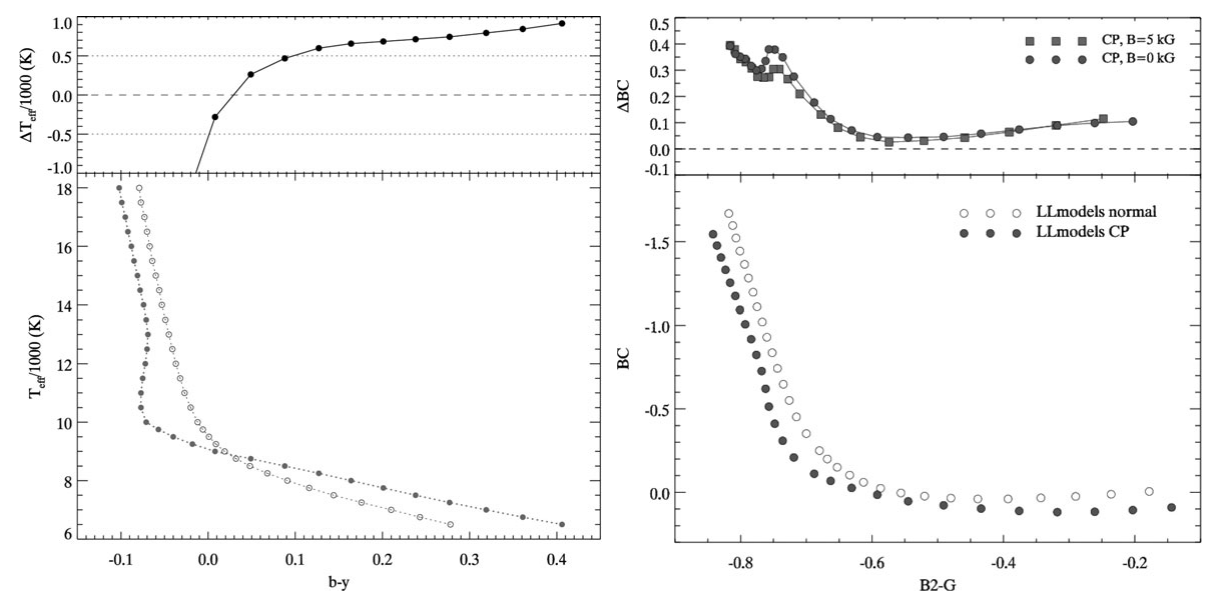

Figure 2. Left panel: theoretical $T_{\text {eff }}$ as a function of Strömgren $b-y$ index for normal (open circles) and CP (filled circles) stars. Right panel: same but for bolometric correction BC as a function of $B_{2}-G$ Genéva index. Upper subplot shows difference in BC of non-magnetic CP and $5 \mathrm{kG}$ magnetic $\mathrm{CP}$ models with respect to models computed with solar abundances.

photometry and RVS spectroscopy will ideally allow the determination of such important parameters as $T_{\text {eff }}, \log (g)$, and metallicity based on specific calibrations and sophisticated algorithms that are presently developed: the final catalog of Gaia observations is scheduled for 2020, after five years of operation and three years of data reduction phase.

Among a billion objects, there will be a certain fraction of CP stars observed. Indeed, depending on spectral classes, $10-30 \%$ of main-sequence B-F stars are CP, and at A0 all slowly rotating stars are CP. The determination of the position of these stars, their parameters, binarity, class of peculiarity etc., would greatly benefit our understanding of $\mathrm{CP}$ phenomena in general and increase the number of potentially interesting objects for detailed research in particular. However, we are faced a difficulty that for stars with moderate or strong peculiarities the standard temperature indicators are inadequate and blind application of the usual photometric calibrations may lead up to $500-1000 \mathrm{~K}$ errors in $T_{\text {eff }}$ determination; see, for example, Shulyak et al., 2008. Generally, there are two reasons why the energy distributions of CP stars differ from those of normal: inhomogeneous horizontal and vertical elements distribution as well as the presence of strong surface magnetic fields. All these modify atmospheric structure and thus lead to abnormal photometric parameters observed for CP stars. As an example, the impact of peculiar opacity on some photometric parameters is shown in Fig. 2, which illustrates theoretically predicted behaviour of some parameters. Theoretical models were computed taking into account characteristic temperature behaviour of abundances of CP stars as derived, for example, in Ryabchikova et al., 2004.

Thus, in order to distinguish between normal and CP stars as observed by Gaia, as well as to derive the type of peculiarity for individual objects, a parametrization of CP stars is needed. This, in turn, requires dedicated model atmospheres to predict their observed parameters. In our investigations we employ the LLMODELS stellar model atmosphere code to compute extensive libraries of high resolution stellar fluxes that are now used for preliminary analysis of Gaia simulated data. LLMODELS is 1-D, LTE model atmosphere code (Shulyak et al., 2004) that treats the bound-bound opacity by direct, line-by-line spectrum synthesis with the fine frequency spacing $\left(10^{5}-10^{6}\right.$ points, $10^{7}$ in parallel mode) thus resolving individual spectral lines. The code does not use any precalculated opacity tables and no assumptions are made about the depth-dependence of the line 

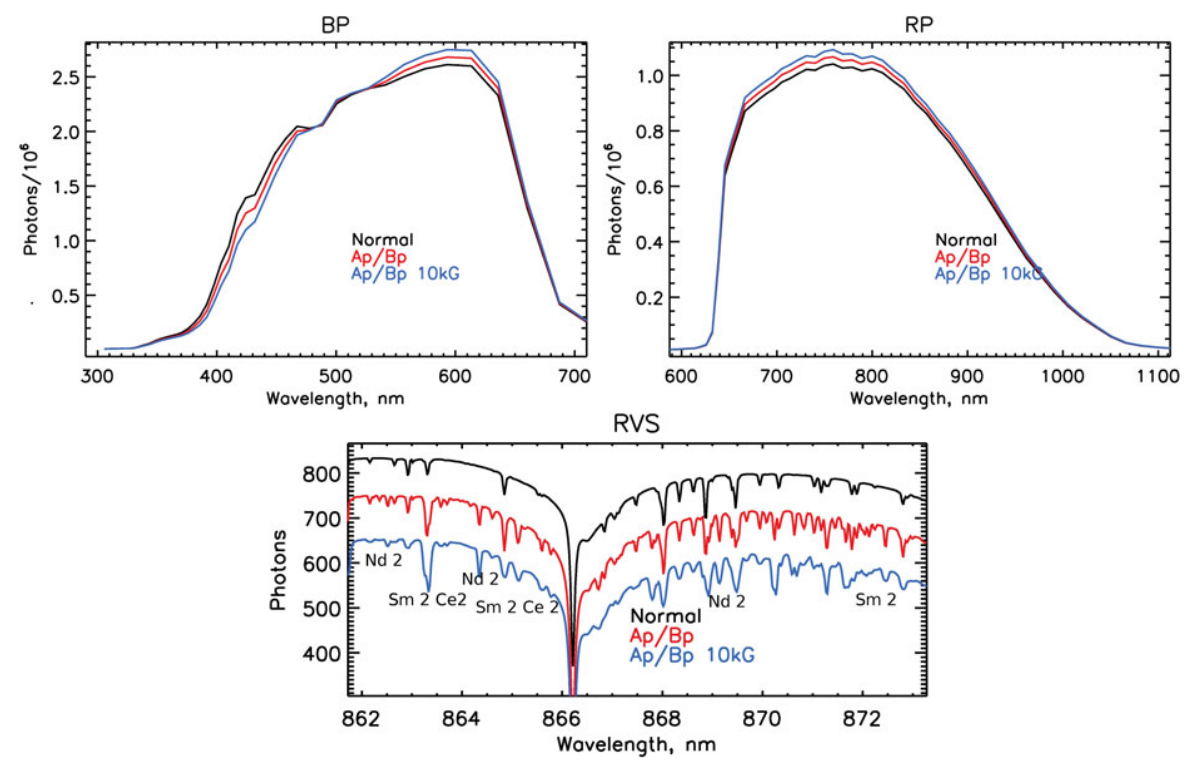

Figure 3. Gaia simulated BP/RP and RVS data for $T_{\text {eff }}=8000 \mathrm{~K}, \log (g)=4.0$ normal and magnetic and non-magnetic Ap stars.

absorption coefficient thus providing a high dynamical range in opacity calculation. This makes it possible to account for the effects of individual non-solar abundance and inhomogeneous vertical distribution of elements. It can also compute models with detailed treatment of anomalous Zeeman splitting, Kochukhov et al., 2005, polarized radiative transfer in all four Stokes parameters Khan \& Shulyak, 2006a, Khan \& Shulyak, 2006b, and magnetohydrostatic equilibrium taken into account, Shulyak et al., 2007, Shulyak et al., 2009a. Chemical abundances and stratification are provided as input parameters for the LLMODELS code and kept constant in the model atmosphere calculation process. This allow us to explore the changes in model structure due to stratification that were extracted directly from observations without modelling the processes that could be responsible for the observed inhomogeneities. Such an empirical modelling can be applied to any CP star for which accurate spectroscopic observations exist; see Kochukhov et al., 2009, Shulyak et al., 2009b and talk JD04-i:5 of this meeting.

Having a suitable code for computing CP star spectra, it is now possible to verify how Gaia sees CP stars. To simulate Gaia data we used the Gaia Object Generator (GOG), a tool originally designed to obtain catalogue data and main database data (including mission final data) for the Gaia satellite. User source input specifications were used, allowing us directly to feed GOG with high resolution fluxes obtained by LLMODELS. Calibration and spectral noise were ignored, mostly because their final models are not yet strictly defined. The model grid of CP stars was computed taking into account characteristic chemistry and effective temperatures of the following types of CP stars: Am/Fm, $\lambda$ Boo, Ap, HgMn, He-weak and He-rich.

As an example, Fig. 3 illustrates the result of simulation of BP, RP, and RVS spectra for a normal $T_{\text {eff }}=8000 \mathrm{~K}, \log (g)=4.0$ star with solar composition, and two Ap stars: non-magnetic, and magnetic with assumed $10 \mathrm{kG}$ surface magnetic field. The energy redistribution due to peculiar abundances and magnetic field is clearly seen, as are abnormally strong lines of REE in the RVS spectrum in case of Ap stars. Thus, there is a chance to use both low resolution spectrophotometry and high resolution spectroscopy 


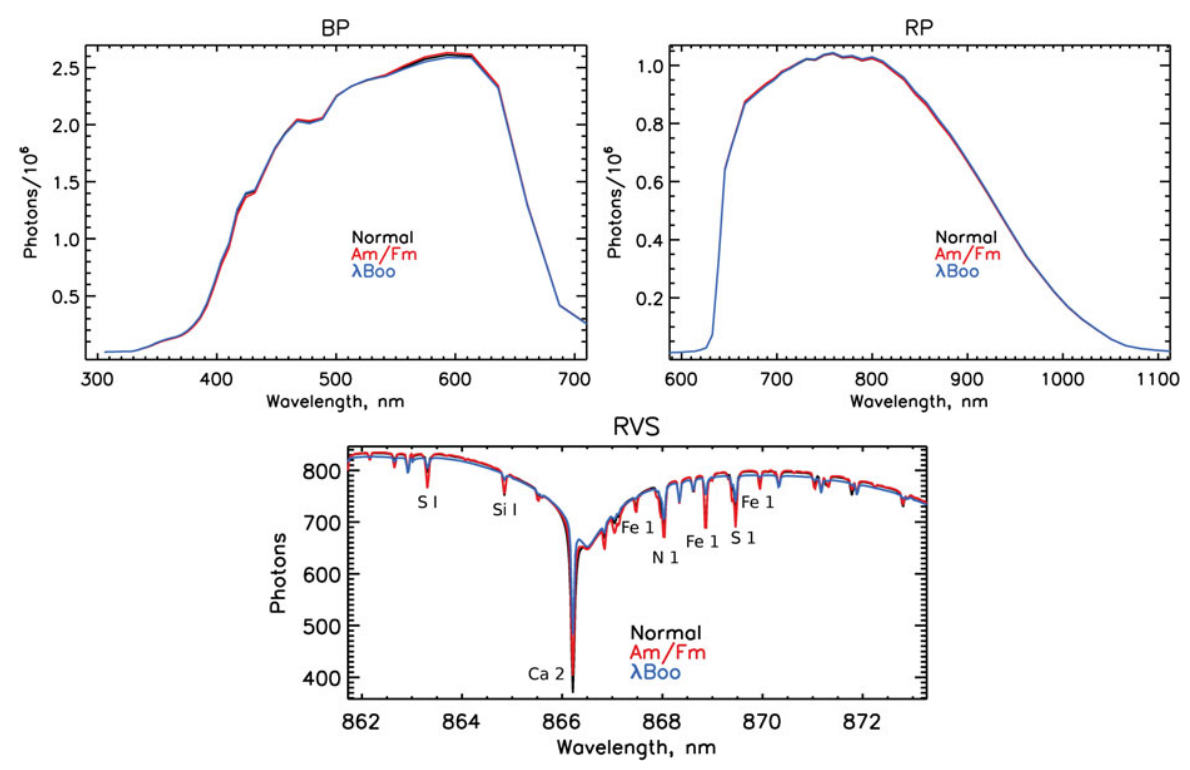

Figure 4. Same as in Fig. 3 but for Am/Fm and $\lambda$ Boo star models.

to verify the type of peculiarity. The example of Am/Fm and $\lambda$ Boo models is presented in Fig. 4.

It follows from our analysis that using $\mathrm{BP} / \mathrm{RP}$ spectra it is only possible to distinguish between Ap and normal stars. Energy distributions of Am/Fm, $\lambda$ Boo, HgMn, He-weak and He-rich stars as seen by Gaia are hardly different from those of normal stars with the same $T_{\text {eff }}$ and $\log (g)$. However, in RVS spectra different types of CP stars are well visible due to the presence of certain spectral features (like deep FeI and CaII lines in case of Am/Fm stars, REE elements in case of Ap stars, MnI/II features in case of HgMn stars, etc.).

Thus, using the BP/RP spectra can most likely help to see CP stars with only strong peculiarities causing substantial energy redistribution from UV to IR and thus easily detectable. On the other hand, analysis of line strength indices or relative parameters (like equivalent widths) in RVS spectra can be applied for all type of CP stars. In addition, after a standard procedure of Gaia's data processing (which will derive fundamental parameters of all observed stars), it will be possible (based on RVS spectra) to derive extended astrophysical parameters like magnetic field strength, signatures of element stratification, starspots, etc.

In the framework of this analysis we carry out calculations of extensive grids of normal and selected CP groups' model atmospheres and fluxes, as well as development, testing and implementation of modified stellar parametrization algorithms as applied to CP stars research.

During its five years' life time, Gaia will observe every object from 40 to 250 times depending upon their individual positions on the sky. The Gaia time sampling is quite irregular, with gaps of typically a month. Still, the probability to recover the periods of strictly periodic signals is high, and this opens a possibility to study a variability of Ap and related stars. The variability amplitude of magnetic CP stars depends on wavelength and is up to $\approx 0.1 \mathrm{mag}$. For stars with $T_{\text {eff }}>10000 \mathrm{~K}$ the amplitude is lower for longer wavelength (decreasing amplitude from $\mathrm{U}$ to $\mathrm{V}$ ), and with $T_{\text {eff }}<10000 \mathrm{~K}$ variability in different filters may be in correlation and thus applying too wide filters may result in 
very weak or no observed variations at all. The characteristic periods are from 0.5 day to decades, and are generally stable.

The preliminary estimate of detected variable Ap stars with Gaia is about 40000 (lower limit) and depends upon the Galaxy model used. This is achieved by the high photometric precision of Gaia in $G$-band $(350-1100 \mathrm{~nm})$ which is $20 \mathrm{mmag}$ at $G=20$ and $\approx 1$ mmag at $10<G<14$. In addition, the application of two separate BP and RP photometers is a good point as it allows to study variability due to energy redistribution from UV to visual and IR.

On the other hand, the detection of roAp variability is a challenging task for Gaia since the characteristic amplitudes are below 0.01 mag and periods are between 6 and 20 minutes, however, there is a possibility to use data from every single CCD of Gaia detector separetely, thus allowing for finer time resolution which is critical for roAp stars. For instance, Mary, 2006 studied a model of roAp star HR 3831 for which they were able to recover three periods assuming stable multiperiodic sinusoidal signal with 16 frequencies (without noise). Later, Varadi et al., 2009 investigated ZZ Ceti stars resulting in $65 \%$ of recovery of a period (multi-periodic nonlinear stable signal with 7 frequencies, with noise at $G=18 \mathrm{mag}$ ). Thus, the goal of using Gaia data in the light of variability research is to detect and classify correctly roAp with some period(s) and amplitude(s) characteristics.

\section{Acknowledgements}

DS would like to acknowledge the support received from the Deutsche Forschungsgemeinschaft (DFG) Research Grant RE1664/7-1 and IAU GA travel grant.

Personal thanks from DS to the GOG WEB administration team.

\section{References}

Babcock, H. W. 1958, ApJS 3, 141

Bruntt, H., North, J. R., Cunha, M., Brandão, I. M., Elkin, V. G., Kurtz, D. W., Davis, J., Bedding, T. R., Jacob, A. P., Owens, S. M., Robertson, J. G., Tango, W. J., Gameiro, J. F., Ireland, M. J., \& Tuthill, P. G. 2008, MNRAS 386, 2039

Cowley, C. R., Hubrig, S., \& González, J. F. 2009, MNRAS 396, 485

Donati, J.-F., Semel, M., Carter, B. D., Rees, D. E., \& Collier Cameron, A. 1997, MNRAS 291, 658

Freyhammer, L. M., Kurtz, D. W., Elkin, V. G., Mathys, G., Savanov, I., Zima, W., Shibahashi, H., \& Sekiguchi, K. 2009, MNRAS 396, 325

Khan, S. A. \& Shulyak, D. V. 2006, A\&A 454, 933

Khan, S. \& Shulyak, D. 2006, A\&SA 448, 1153

Khokhlova, V. L., Vasilchenko, D. V., Stepanov, V. V., \& Tsymbal, V. V. 1997, Astron. Lett. 23,465

Kholtygin, A. F., Chountonov G. A., Fabrika, S.N. et al. 2007, in Physics of Magnetic Stars, Proc. Intern. Conf. held in the Special Astrophysical Observatory, Russia, August 28-31, 2006, Eds. I. I. Romanyuk and D. O. Kudryavtsev, p. 262

Kholtygin, A. F. 2008, in Clumping in Hot Star Winds, W.-R. Hamann, A. Feldmeier \& L. M. Oskinova, eds., Potsdam: Univ.-Verl., p. 167

Kochukhov, O., Shulyak, D., Ryabchikova, T. 2009, A\&A 499, 851

Kochukhov, O. 2008, A\& $A$ 483, 557

Kochukhov, O., Tsymbal, V., Ryabchikova, T., Makaganyk, V., \& Bagnulo, S. 2006, A\&\&A 460, 831

Kochukhov, O. 2006, A\&SA 446, 1051

Kochukhov, O., Khan, S., \& Shulyak, D. 2005, A\& A 433, 671

Kochukhov, O., Drake, N. A., Piskunov, N., \& de la Reza, R. 2004, A\&\&A 424, 935 
Kurtz, D. W. 1978, IBVS, 1436, 1

Lanz, T. 1993, ASP-CS 44, 60

Lüftinger, T., Fröhlich, H.-E., Petit, P., Aurière, M., Weiss, W., Nesvacil, N., Gruberbauer, M., Shulyak, D., Alecian, E., Catala, C., Donati., J.-F., Kochukhov, O., \& Roudier, T. 2009, $A \mathscr{E} A$, submitted

Michaud, G. 1970, ApJ 160, 641

Mikulášek, Z., Krtička, J., Henry, G. W., Zverko, J., Žižåovský, J., Bohlender, D., Romanyuk, I. I., Janík, J., Božić, H., Korčáková, D., Zejda, M., Iliev, I. K., Škoda, P., Šlechta, M., Gráf, T., Netolický, M., \& Ceniga, M. 2008, A\&\&A 485, 585

Mary, D. L. 2006, $A \& A 452,715$

North P. 1993, ASP-CS 44, 577

Pickering, E. C. \& Maury, A. C., 1897, Annals of Harvard College Observatory 28, 1

Preston, G. W. 1974, ARAA 12, 257

Przybilla, N., Nieva, M. F., Tillich, A., Heber, U., Butler, K., \& Brown, W. R. 2008, A $\& A 488$, 51

Ryabchikova, T., Kochukhov, O., \& Bagnulo, S. 2008, A\& A 480, 811

Ryabchikova, T., Ryabtsev, A., Kochukhov, O., \& Bagnulo, S. 2006, A\&A 456, 329

Ryabchikova, T., Leone, F., \& Kochukhov, O. 2005, A\&SA 438, 973

Ryabchikova, T., Nesvacil, N., Weiss, W. W., Kochukhov, O., \& Stütz, Ch. 2004, A $\& A$ 423, 705

Ryabchikova, T., Piskunov, N., Kochukhov, O. et al. 2002, A\&\&A 384, 545

Shulyak, D., Kochukhov, O., Valyavin, G., Lee, B.-C., Galazutdinov, G., Kim, K.-M., Han, I., \& Burlakova, T. 2009, A\&\&A, submitted

Shulyak, D., Ryabchikova, T., Mashonkina, L., \& Kochukhov, O. 2009, A\&3A 499, 879

Shulyak, D., Kochukhov, O., \& Khan, S. 2008, A\&A 487, 689

Shulyak, D., Valyavin, G., Kochukhov, O., Lee, B.-C., Galazutdinov, G., Kim, K.-M., Han, I., Burlakova, T., Tsymbal, V., \& Lyashko, D. 2007, A\&A 464, 1089

Shulyak, D., Tsymbal, V., Ryabchikova, T., Stütz Ch., \& Weiss, W. W. 2004, A\&A 428, 993

Sokolov, N. A. 2008, MNRAS 385, 1

Varadi, M., Eyer, L., Jordan, S., Mowlavi, N., \& Koester, D. 2009, in Stellar Pulsation: challenges for theory and observation, AIP Conference Series, vol 1170, 330

Wade, G. A., Donati, J.-F., Landstreet, J. D., \& Shorlin, S. L. S. 2000, MNRAS 313, 823

Woszczyk, A. \& Jasinski, M. 1980, AcA 30, 331 